PROF. SANDRA W. JACOBSON (Orcid ID : 0000-0002-9768-2671)

PROF. PETER HAMMOND (Orcid ID : 0000-0003-3208-4459)

Article type : Original Research Article

\title{
Facial curvature detects and explicates ethnic differences in effects of prenatal alcohol exposure
}

Michael Suttie, M.Sc. ${ }^{1,2}$, Leah Wetherill, M.Sc. ${ }^{3}$, Sandra W. Jacobson, Ph.D. ${ }^{4,5}$, Joseph L. Jacobson, Ph.D. ${ }^{4,5}$, H. Eugene Hoyme M.D. ${ }^{6}$, Elizabeth R. Sowell Ph.D. ${ }^{7}$, Claire Coles Ph.D. ${ }^{8}$, Jeffrey R. Wozniak M.D. ${ }^{9}$, Edward P. Riley Ph.D. ${ }^{10}$, Kenneth L. Jones M.D. ${ }^{11}$, Tatiana Foroud, Ph.D. ${ }^{3}$, Peter Hammond, Ph.D. ${ }^{1,2}$ and the CIFASD

${ }^{1}$ Nuffield Department of Obstetrics and Gynaecology, ${ }^{2}$ Big Data Institute, University of Oxford, Oxford, UK; ${ }^{3}$ Department of Medical and Molecular Genetics, Indiana University School of Medicine, Indianapolis, USA; ${ }^{4}$ Wayne State University School of Medicine, Department of Psychiatry and Behavioral Neurosciences, Detroit, USA; , ${ }^{5}$ Departments of Human Biology and of Psychiatry and Mental Health, University of Cape Town Faculty of Health Sciences, Cape Town, South Africa; ${ }^{6}$ Sanford Research and Department of Pediatrics, Sanford School of Medicine, University of South Dakota, Sioux Falls, USA; ${ }^{7}$ Developmental Cognitive Neuroimaging Laboratory Children's Hospital Los Angeles, USA; ${ }^{8}$ Department of Psychiatry and Behavioral Sciences, Emory University School of Medicine, Atlanta, USA; ${ }^{9}$ Department of Psychiatry, University of Minnesota, Minneapolis, USA; ${ }^{10}$ Department of Psychology, San Diego State University, San Diego, USA; ${ }^{11}$ Department of Pediatrics, School of Medicine, UCSD, San Diego, USA.

This is the author's manuscript of the article published in final edited form as:

Suttie, M., Wetherill, L., Jacobson, S. W., Jacobson, J. L., Hoyme, H. E., Sowell, E. R., Coles, C., Wozniak, J. R., Riley, E. P., Jones, K. L., Foroud, T., Hammond, P. and The CIFASD (), Facial curvature detects and explicates ethnic differences in effects of prenatal alcohol exposure. Alcohol Clin Exp Res. Accepted Author Manuscript. http://dx.doi.org/10.1111/acer.13429 


\section{Correspondence:}

Professor Peter Hammond,

Big Data Institute \& Nuffield Department of Obstetrics and Gynaecology,

University of Oxford, Oxford, OX3 7FZ, UK

Tel: +44 (0) $1865221004 \quad$ Fax: +44 (0) 1865769141

Email: peter.hammond@obs-gyn.ox.ac.uk

\section{Sources of Support:}

This international collaborative study was completed in conjunction with the Collaborative Initiative on Fetal Alcohol Spectrum Disorders (CIFASD) and is funded by NIH/National Institute on Alcohol Abuse and Alcoholism: U01AA014809 (T.F. and P.H.); U24AA014815 (K.L.J.); U01AA017122 (E.R.S.); U24AA014811 (E.J.R.); the Cape Town Longitudinal Cohort Study, by R01AA09524, U01AA014790, R01AA016781 (S.W.J.). Additional information about CIFASD can be found at www.cifasd.org.

\section{ABSTRACT}

Background: Our objective is to help clinicians detect the facial effects of prenatal alcohol exposure (PAE) by developing computer based tools for screening facial form.

Methods: All 415 individuals considered were evaluated by expert dysmorphologists and categorized as 1) healthy control (HC), 2) fetal alcohol syndrome (FAS) or 3) heavily prenatally alcohol exposed (HE) but not clinically diagnosable as FAS. 3D facial photographs were used to build models of facial form to support discrimination studies. Surface curvature based delineations of facial form were introduced.

This article is protected by copyright. All rights reserved. 


\section{Results:}

a. Facial growth in FAS, HE and control subgroups is similar in both cohorts.

b. Cohort consistency of agreement between clinical diagnosis and HC-FAS facial form classification is lower for mid-line facial regions and higher for non-mid-line regions.

c. Specific HC-FAS differences within and between the cohorts include:

for HC, a smoother philtrum in Cape Coloured individuals;

for FAS, a smoother philtrum in Caucasians;

for control-FAS philtrum difference, greater homogeneity in Caucasians;

for control-FAS face difference, greater homogeneity in Cape Coloured individuals;

d. Curvature changes in facial profile induced by prenatal alcohol exposure are more homogeneous and greater in Cape Coloureds than in Caucasians.

e. The Caucasian HE subset divides into clusters with control-like and FAS-like facial dysmorphism. The Cape Coloured HE subset is similarly divided for non-mid-line facial regions but not clearly for mid-line structures.

f. The Cape Coloured HE subset with control-like facial dysmorphism shows orbital hypertelorism.

This article is protected by copyright. All rights reserved. 


\section{Conclusions:}

Facial curvature assists the recognition of the effects of prenatal alcohol exposure and helps explain why different facial regions result in inconsistent control-FAS discrimination rates in disparate ethnic groups. Heavy prenatal alcohol exposure can give rise to orbital hypertelorism, supporting a long-standing suggestion that prenatal alcohol exposure at a particular time causes increased separation of the brain hemispheres with a concomitant increase in orbital separation.

Key words: facial curvature; facial dysmorphism; fetal alcohol spectrum disorders; 3D face analysis.

Abbreviations: $D S M=$ dense surface model; FAS=fetal alcohol syndrome; FASD=fetal alcohol spectrum disorder; PFAS=partial fetal alcohol syndrome; $\mathrm{HE}=$ heavy alcohol exposure but not diagnosable clinically as FAS or PFAS; HC=healthy control.

\section{INTRODUCTION}

Prenatal alcohol exposure causes a continuum of effects. Fetal alcohol syndrome (FAS), at the more severe end of the phenotype, affects growth, face shape and neurobehavior (Jones et al, 2006; Hoyme et al, 20015; Jones and Smith, 1973). Fetal alcohol spectrum disorders (FASDs) include FAS and other pathologies arising from prenatal alcohol exposure. Several studies have explored image-based recognition of FAS facial features (Moore et al,

This article is protected by copyright. All rights reserved. 
2001, 2002, 2007; Mutsvangwa and Douglas, 2007; Fang et al, 2008; Mutsvangwa et al, 2010; Douglas and Mutsvangwa, 2010; Foroud et al, 2012; Astley and Clarren, 1996, 2000; Klingenberg et al, 2010; Suttie et al, 2013).

In a previous study of South African Cape Coloured children (Suttie et al, 2013), we used dense surface models (Bhuiyan et al, 2006; Cox-Brinkman et al, 2007; Hammond, 2007; Hammond et al, 2004, 2005, 2008, 2011; Hutton et al, 2003; Kasperavičiūtė et al, 2011; Tassabehji et al, 2006; Tobin et al, 2008; Lipinksi et al, 2012) to induce HC-FAS discrimination and established strong agreement between classification based on face alone and clinical diagnosis of FAS. The more heterogeneous phenotype of individuals not clinically diagnosable but heavily alcohol exposed (HE) caused us to employ a clustering technique (Sturn et al, 2002), signature graph analysis (Hammond et al, 2012; Hammond and Suttie, 2012), which normalizes differences in facial form against healthy controls (HC) and links individuals with similar facial dysmorphism. Signature graph analysis partitioned the Cape HE group into those having facial dysmorphism more FAS-like and those more control-like with the former performing less well on psychometric tests than did the latter (Suttie et al, 2013).

In this study, we compare the Cape Coloured cohort studied previously and a Caucasian cohort recruited within the CIFASD consortium. In particular, we demonstrate how facial curvature, not previously considered in FASDs, can assist the recognition of the facial effects of prenatal alcohol exposure and help explain inconsistent control-FAS discrimination rates in different facial regions for the two ethnic groups. We also demonstrate that heavy prenatal alcohol exposure can give rise to orbital hypertelorism in individuals who are not clinically diagnosable. This intriguing result provides supporting This article is protected by copyright. All rights reserved. 
evidence for a long-standing conjecture that prenatal alcohol exposure at a particular time, e.g., human gestation week 4, can cause increased separation of the brain hemispheres with a concomitant increase in orbital separation (Parnell et al, 2009; Sulik et al, 1986).

\section{MATERIALS AND METHODS}

\section{Participants}

The 166 South African participants aged 6.8 to 16.2 years were recruited from the Cape Coloured (mixed ancestry) community (Jacobson et al, 2008, 2011) in Cape Town, South Africa, where the incidence of heavy alcohol use during pregnancy and of FAS are among the highest in the world (May et al, 2016a; Roozen et al, 2016). A Caucasian cohort of 249 participants aged 3 to 18 years was recruited by the CIFASD consortium in several countries (207 in USA; 42 in Europe). We excluded African-American, Native-American, Asian and Hispanic recruits because of the paucity of FAS diagnoses in those subgroups. The PFAS diagnostic category was not used in the Caucasian cohort. The Cape Coloured:Caucasian diagnostic subgroup sizes were as follows: control (69:141); FAS (22:35); HE (75:73). Appropriate consent was obtained for all participants (written informed consent from mothers and oral assent from children) according to IRB-approved criteria.

\section{Clinical assessment}

Supplementary Table ST1 summarizes criteria we have used to characterize FAS and partial FAS (PFAS) and differentiate them from non-exposed healthy controls ( $\mathrm{HC}$ ) and nonsyndromal heavy alcohol exposure (HE) where the FAS/PFAS criteria are not fully met (CDC, 2004). Each child was examined for growth deficits and FAS facial features independently by This article is protected by copyright. All rights reserved. 
one or more expert dysmorphologists blinded to prenatal alcohol exposure history, using a standard protocol (Hoyme et al, 2005) employing the Astley Lip-Philtrum Guide (Astley and Clarren, 2001) for assessing philtrum and upper lip vermilion. Palpebral fissure length was measured manually. Individuals with known genetic disorders were excluded. Where multiple dysmorphology experts examined a child, a final FASD categorizatio was agreed by consensus. Offspring of women who reported abstaining or low consumption were designated as controls, unless they met FAS criteria.

\section{Face analysis}

Image capture, preparation and building of shape models

3D facial images were captured with commercial photogrammetric cameras. One individual (MS) landmarked each image at 24 validated locations (Gwilliam et al, 2006) (Fig. 1). The landmarks were used to (Procrustes) align the face surfaces, induce a dense correspondence of surface points and determine average surfaces of subgroups. For the face, and seven smaller facial regions (Fig. 1), dense surface models (DSM) were computed as the set of principal component analysis (PCA) modes arising from the displacement differences of the constituent faces from their overall average. Landmarking, DSM building and shape analysis were undertaken using software developed in-house (Hammond, 2007; Hammond and Suttie, 2012).

This article is protected by copyright. All rights reserved. 
Clinical categorization and face based classification

The agreement of clinical categorization and discrimination using DSM representation of facial form was estimated from a standard multi-folded supervised learning strategy employing 20 random 90\%-10\% training-unseen test pairs of subject subsets (stratified with respect to affected/unaffected status) using receiver operating characteristic curve analysis. Each estimate of agreement corresponds to the probability of correctly classifying two individuals, one taken randomly from each of the subgroups being compared. Closest mean classification labels members of two groups by the name of the group whose mean is most similar. For linear discriminant analysis, the goal is a linear combination of PCA modes that exhibits the largest difference in the subgroup means relative to the within-group variance. Support vector machines, or large margin classifiers, focus on individual cases in the overlap of the subgroups to be classified that help to define a separating surface with largest margin between the subgroups. As well as the full face, patches of the face were also considered in isolation: eyes, philtrum, mandible, nose, malar region, upper lip vermilion and mid-line profile (Fig. 1). Patches focussing on the philtrum, upper lip vermilion and malar only regions were not included in the previous Cape Coloured study. Besides the "supervised learning" pattern-matching algorithms mentioned above, we also used signature graph "unsupervised" cluster analysis as described elsewhere (Hammond et al, 2012; Hammond and Suttie, 2012; Suttie et al, 2013).

This article is protected by copyright. All rights reserved. 
Displacement face signatures and color-coded heat maps of average and individual faces

Each individual was matched with 35 age-contiguous same ethnicity controls whose mean age most closely matched their age. The displacements of densely corresponded points on an individual's face orthogonal to its surface were normalized against corresponding displacements on faces of matched controls to produce a displacement face signature (Hammond et al., 2012).

Individual displacement face signatures were heat mapped to visualize normalized shape differences from matched controls using a red-green-blue colour scale at min-0-max units of standard deviation for appropriate minima and maxima (-2SD and +2SD unless otherwise stated). Thus, for displacement normal to the face surface, the red/green/blue spectrum corresponded to contraction/coincidence/expansion of the surface being compared. Analogous processes produced signatures for surface shape displacement parallel to lateral, vertical, and depth axes. The signature weight of an individual face or face region (square root of sum of squared normalized differences for all densely corresponded points) reflected the deviation of a subject from matched controls and thus estimates the facial dysmorphism of an individual in terms of surface displacement (Hammond et al, 2012).

A face signature graph is constructed using face signatures as vertices or nodes and a directed edge links each vertex to its most "similar" signature. An alternative "colored" form of a face signature paints vertices to reflect a diagnostic or other labelling such as controlFAS or Caucasian-Cape Coloured dichotomy. Finally, a "colored" signature graph can be "collapsed" to a simplified form where connected vertices of the same color are combined into a super-vertex while preserving connectivity to differently colored vertices. An entropyThis article is protected by copyright. All rights reserved. 
like measure, dispersion index, is used to summarize the associated clustering or dispersion of the members of each labelled subgroup (Hammond et al, 2012). The lower the dispersion index of a labelled subgroup, the stronger is the similarity or homogeneity of the facial dysmorphism of subcluster members. A dispersion index of 1.00 means that a subgroup's members are perfectly dispersed into singleton subsets and so do not cluster at all because they lack homogeneity. In contrast, a dispersion factor of 0 means the displacement dysmorphism of a subgroup is perfectly homogeneous and its members connect only to each other. If there are just two classes of nodes in a signature graph (e.g., control/FAS for same cohort or Cape/Caucasian for same diagnosis), a pair of dispersion indices encapsulates the relative homogeneity of each class and their degree of mixing or relative similarity in terms of normalised difference from matched controls. All signature graphs were drawn using the open source software GraphViz (version 2.26, www.graphviz.org; Ellson et al, 2002).

\section{Curvature visualisation and quantification}

Curvature of the face surface was defined relative to the surface mesh of points in the 3D photograph. The algorithm employed is a standard one from the VTK library (www.vtk.org) and determines mean curvature at a mesh point in terms of the angles subtended by surface facets (here, triangles) that are adjacent at the point. It can be delineated as a heat map, with convex/prominent features, such as the nose, colored blue and concave grooves or inward creases, such as the philtrum, colored red. Small, isolated deeply colored facets are caused by noisy components (spikes and troughs) generated during image capture.

This article is protected by copyright. All rights reserved. 
Curvature can also be determined with respect to an axis orthogonal to a plane in which the curvature falls. Two specific axial curvatures are particularly useful in studying the shape of the philtrum groove: some individuals have a deep vertical groove and others have little in the way of a vertical groove but have a curl-like shape (similar to a ski-jump). We use the terminology "curl" and "groove" to distinguish between them. The facial landmarks left and right exocanthi were used to define a horizontal axis relative to the face surface and with the thumb in the direction of the axis the back of the fingers follow the curl we are delineating. Analogously, an axis defined by landmarks subnasale and upper lip centre identifies curvature forming the typical "vertical" groove of the philtrum. The independence of curl and groove curvature is demonstrated in Table 1 by four specifically selected examples. Groove curvature is particularly effective in delineating the philtrum pillars. The examples of Table 1 show how philtrum flatness in the form of low curl or low groove is demarcated by a blue patch in the middle of the philtrum surface. A signature representing normalised curvature difference across a face surface and curvature signature graphs can be defined analogously as for surface displacement. The significance of localised curvature is delineated by normalization with respect to age-ethnicity matched controls at each of the densely corresponded surface points in the DSM.

A curvature heat map, since it ignores displacement difference, is often much better at delineating philtrum smoothness. A displacement heat map of an FAS face compared to matched controls, since it reflects size as well as shape, may display strong red region across the entire face delineating "inward" displacement that swamps smaller "outward" displacement difference of a smooth philtrum. Examples illustrating this appear in supplementary information (Fig. SF1).

This article is protected by copyright. All rights reserved. 
Fig. 2 contains the philtrum groove curvature signatures of all FAS individuals, both Cape Coloured and Caucasian. The greater homogeneity of the philtrum groove signatures of Caucasians compared to Cape Coloured individuals is clearly seen. This is reflected in the collapsed groove signature graph (with a large node containing 32 Caucasian FAS individuals) and associated dispersion indices of 0.54 and 0.10 respectively for Cape and Caucasian subsets, emphasising the Caucasian homogeneity of prenatal alcohol exposure induced philtrum groove differences.

\section{RESULTS}

Table 2 summarises HC, FAS and HE subset comparisons of the Cape-Coloured and Caucasian cohorts for variation in age, BMI, palpebral fissure length and lip-philtrum scores. The only difference found was significantly greater BMI in the Caucasian HE subset compared to the Cape Coloured HE subset $(p<0.01)$.

The facial growth of HE individuals and controls was close in both cohorts. For FAS, significant reduction in facial growth occurred in both cohorts without significant difference between them.

Separate cohort specific dense surface models of the face for HC, FAS and HE individuals were constructed. In both models, PCA1 represents overall facial growth. The raw PCA1 scores (Figs. $3 \mathrm{~A}$ and $3 \mathrm{~B}$ ) show facial growth in the HE subgroups to be control-like but consistently marginally reduced. In order to avoid HE individuals influencing the comparison of $\mathrm{HC}$ and FAS growth, they were omitted from a further pair of cohort specific dense surface models for the face surface. For each model, PCA1, once again representing facial growth, was normalised for age and ethnicity (Supplementary Fig. SF2). Comparison of the 
box plots for these age-ethnicity corrected PCA values (Fig. 4A) confirms that although facial growth in individuals with FAS was very significantly subdued compared to controls in both the Cape Coloured $\left(p<10^{-12}\right)$ and Caucasian $\left(p<10^{-16}\right)$ cohorts, there was no significant difference for FAS between the cohorts $(p=0.32)$.

\section{Consistency between cohorts for agreement of face based control-FAS classification with} clinical diagnosis was lower in mid-line regions (nose, profile, upper lip vermilion and philtrum) and higher in non-mid-line regions (face, eyes, malar and mandible).

Three classification algorithms (closest mean, linear discriminant analysis and support vector machines) were used previously to test agreement of control-FAS discrimination based only on face surface displacement with clinical categorization of the Cape Coloured cohort (Suttie et al, 2013). Table T3 contains the rates of discrimination for both cohorts and all facial regions tested. Figure $4 B$, a simplified summary of the table, plots the average performance of the three algorithms, Cape Coloured against Caucasian, for each facial region. Cohort consistency of classification was strongest for non-mid-line facial regions: face, eyes, mandible and malar region. In contrast, for mid-line regions (nose, philtrum, profile and upper lip vermilion) there was less consistency. In the Cape cohort, the classic regions of philtrum and upper lip vermilion regions were weaker than the less studied malar region for control-FAS discrimination. For the Caucasian cohort, the face, eyes, nose and malar region performed well, whereas the profile was weaker. In the following, we attempt to explain some of these discrepancies between the two cohorts.

This article is protected by copyright. All rights reserved. 


\section{Explaining Cape-Caucasian discrepancies in control-FAS classification for the philtrum:}

\section{a) for controls, the philtrum was smoother in Cape individuals than in Caucasians}

Comparison of mean philtrum shape suggested that the upper half of the Cape control philtrum was flatter than the Caucasian control philtrum (Figs. 5A \& 5F). A morph between the two confirmed this (supplementary video Control_Cape_Cauc_philtrum_morph.avi). Normalisation against age-matched (and not the usual ethnicity matched) Caucasian controls delineated greater concavity immediately close to the subnasale of the Cape Coloured mean (superior red region in Fig. 5B) and greater flatness of the philtrum groove (blue in both curl and groove heat maps, Figs. 5B \& 5C). This suggested that on average the (upper) philtrum is smoother in Cape Coloured controls than in Caucasian controls.

\section{b) for FAS, the philtrum was smoother in Caucasians than in Cape individuals}

The heat maps in the last column of Fig. 5 delineated normalisation of average groove curvature employing the more usual matched ethnicity. They highlighted more extensive flatness of the philtrum groove in Caucasian FAS compared to that of Cape FAS (blue patches in Figs. 5E \& 5H). Thus, the philtrum smoothing effect of prenatal alcohol exposure appeared to be stronger in the Caucasian cohort. Face signature normalises shape differences with respect to ethnically matched controls, so DSMs for the combined cohorts can generate a signature graph for all FAS individuals or for all controls, as well as their collapsed forms and associated dispersion indices. Table 4 contains Caucasian-Cape dispersion indices for Controls and FAS individuals for face and philtrum. Examples of associated signature graphs are shown in Supplementary Figures SF3 and SF4.

This article is protected by copyright. All rights reserved. 
c) Caucasian FAS-Control philtrum difference was more homogeneous than in Cape individuals

In the FAS row of Table 4, the second pair of emboldened dispersion indices ( 0.56 and $\mathbf{0 . 0 7})$ and their counterparts in the CONTROLS row (0.69 and 0.19) suggested that the effect of prenatal alcohol exposure was to reduce dispersion in the Caucasian FAS collapsed signature graph, i.e., to make the philtrum groove curvature more homogeneous in its difference from controls. In contrast, the corresponding Cape Coloured indices for controls ( 0.79 and 0.59 ) hardly altered for FAS (0.75 and 0.55). Indeed, in Fig. 2, the greater homogeneity of the Caucasian FAS philtrum groove signatures was well delineated in the individual heat maps and was further corroborated in the collapsed signature graph by a large sub-cluster of 32 of 35 Caucasian individuals with FAS and a correspondingly low dispersion index.

Results $a, b$ and c were likely to enhance control-FAS classification for the Caucasian philtrum.

Changes in curvature of the mid-face profile induced by prenatal alcohol exposure were more homogeneous and significantly greater in Cape Coloureds than in Caucasians. These factors were likely to increase control-FAS discrimination for the Cape Coloured mid-face profile.

The Caucasian/Cape control dispersion coefficients for a joint curl signature graph for the mid-face profile, $0.28 \pm 0.01 / 0.73 \pm 0.01$, suggest that normalised curvature of the facial profile is more homogeneous in Caucasians than in Cape Coloureds. For individuals with a FAS diagnosis, the corresponding Caucasian/Cape dispersion coefficients are This article is protected by copyright. All rights reserved. 
$0.48 \pm 0.03 / 0.31 \pm 0.02$. These data suggest that the effect of prenatal alcohol exposure on the mid-face profile results in a more uniform difference from controls in Cape Coloureds than in Caucasians. The associated collapsed curl signature graphs corroborate these results (Supplementary Fig. SF5) and, furthermore, the degree of altered curvature induced by the prenatal alcohol exposure (as measured by the log transformation of curl signature weight) is significantly greater in Cape Coloureds than in Caucasians (mean of Cape Coloured individuals: 1.49 ; mean of Caucasians: $1.42 ; \mathrm{p}<0.05)$. These factors are likely to enhance control-FAS discrimination of the mid-line profile for Cape Coloureds compared to Caucasians.

\section{As for the Cape Coloured cohort, the Caucasian HE subset was partitioned in a displacement signature graph into subclusters with control-like or FAS-like facial dysmorphism.}

In the previous Cape-Coloured cohort study (Suttie et al., 2013), we showed how a face signature graph partitioned the HE subset into FAS/PFAS-like and control like dysmorphism subgroups with corresponding FAS/PFAS-like and control-like cognitive impairment. Because there is no PFAS subcategory in the Caucasian cohort, we retested the original CapeColoured finding omitting those individuals with a PFAS clinical categorization. Reassuringly, the resulting $\mathrm{HE}$ partition was identical (Fig. 6A). The analogous signature graph for combined Caucasian FAS and HE subgroups (Fig. 6B) produced a similar partition of the Caucasian HE subgroup. However, different batteries of cognitive measures were used to

This article is protected by copyright. All rights reserved. 
assess the Caucasian cohort at different stages of the CIFASD consortium. Therefore, without common measures throughout, we could not determine if the FAS-like and controllike facial displacement differences corresponded to differences in cognitive impairment as we did with the Cape Coloured cohort.

The Cape HE subset was partitioned into similar FAS-like (HE-FAS) and control-like (HE-HC) clusters for normalised displacement of non-mid-line facial regions: periorbital, mandibular and malar; but, not for mid-line structures: nose, upper lip, philtrum and profile.

The collapsed signature graphs of the combined HE and FAS subsets of each cohort for different facial regions are shown in the supplementary data (Supplementary Figure SF6). For Cape Coloured individuals, the partitioning of HE into FAS-like dysmorphism and controllike dysmorphism is clear for the eyes, mandible and malar region. For the mid-line regions (nose, upper lip, philtrum and profile) there is no clear cut partitioning.

The Cape HE-HC subgroup derived from the face displacement signature graph displayed orbital hypertelorism $(p<0.01$ Independent-samples 2 -tailed t-test not assuming equal variance in SPSS 23).

A signature heat map of horizontal $\mathrm{x}$-axis displacement for the mean of the HE-HC subset of Cape Coloured individuals shows clear outward displacement of the left and right periorbital regions (Fig. 7A). Orbital hypertelorism is quantitatively confirmed by significantly increased inner canthal and outer canthal separation as well as greater inter-pupillary separation, the latter being computed following the addition of pupillary landmarks for just the Cape Coloured HC and HE-HC subsets (Figs. 7B to 7D).

This article is protected by copyright. All rights reserved. 


\section{Conclusions}

The diagnosis of FAS is multi-faceted and difficult. Recognition of the associated facial features, shortened palpebral fissures, smooth philtrum and thin upper lip vermilion, is an essential component of diagnosis. Even more challenging is the situation where the classic facial characteristics are not all present or are too subtle for the inexperienced or unaided eye to discern. An important aspect of our approach is the development of objective techniques to assist the screening of faces for features associated with prenatal alcohol exposure and to identify small, subtle differences and how they relate to ethnic background across the FASD spectrum.

Given that some clinicians are relatively confident in diagnosing FAS, it might seem odd to test our classification on FAS. It is important to test any face screening tools on their capacity to discriminate previously diagnosed cases of FAS from healthy controls before establishing their efficacy in identifying so-called HE individuals with confirmed heavy prenatal alcohol exposure for whom a diagnosis of FAS is not clinically possible. In this, and a previous, study on a Cape-Coloured cohort, we have demonstrated that dense surface modelling techniques applied to overall facial form are highly effective in control-FAS discrimination. However, when similar analysis was made of localised regions of the face, the discrimination efficacy was more variable depending on the cohort and the region considered. Interestingly, control-FAS discrimination was less consistent in the two cohorts for mid-line facial regions and more consistent for non-mid-line regions. Possible explanations for this are that prenatal alcohol exposure has a more variable effect on midline development or that some ethnic groups are differentially susceptible to mid-line effects of prenatal alcohol exposure.

This article is protected by copyright. All rights reserved. 
It was somewhat surprising to see that the malar region, despite being relatively featureless compared to the mid-face, was very discriminating for both cohorts. Indeed, it was almost as discriminating as the full face. Of course, judging smoothness of the philtrum or thinness of the upper lip by eye is easier than identifying changes in the feature rich facial profile or the feature poor malar region. For clinical use, the philtrum and upper lip are more convenient markers but with computational support for image-based analysis, other regions of the face may be equally, or even more, informative.

The philtrum was more discriminating between controls and individuals with FAS for the Caucasian cohort but the reverse held for the Cape Coloured cohort. These discrepancies in discrimination performance were unexpected and so to understand them better, curvature analysis of the face was introduced. Up to that point, the approach had been to emphasise localised differences in size normal to the face surface. Given the importance of flatness of the philtrum in the recognition of the FAS facial phenotype, it was natural to consider curvature. The notion of curvature of the face surface is quite intuitive and a simple extension of general curvature relative to the surface normal enabled more axial interpretations such as curl and groove to be introduced to emphasise particular undulations of the philtrum surface.

It appears that the poorer discrimination of the philtrum region for the CapeColoured cohort may be partly explained by its relative flatness proximal to the nostrils in controls whereas the Caucasian control philtrum tends to be more grooved throughout its length. More importantly, the flattening of the philtrum groove in the Cape-Coloured FAS subgroup as a result of prenatal alcohol exposure appears to be weaker than in the Caucasian FAS subgroup.

This article is protected by copyright. All rights reserved. 
The homogeneity of the shape difference of the mid-face profile of Caucasian controls was shown to be greater than that of Cape Coloured controls. This may be due to the latter's ethnic ad-mixture (de Wit et al., 2010) of Khoisan (32\%-43\%), Bantu speaking African (20\%-36\%), European (21\%-28\%) and Asian (9\%-11\%) ancestries. Nevertheless, the effect of prenatal alcohol exposure on the mid-face profile in Cape Coloured individuals with a diagnosis of FAS resulted in greater and more homogeneous dysmorphism, which is likely to assist control-FAS discrimination and explain the higher discrimination rate for the Cape Coloured profile compared to Caucasians.

The ability of a signature graph based on similarity of facial dysmorphism to partition the Cape-Coloured HE subgroup into disjoint subgroups reflecting cognitive impairment is potentially a very useful screening device. This is particularly so for the identification of individuals who may have been exposed prenatally to alcohol but whose facial phenotype does not fit the classic one for FAS. A similar partition of the Caucasian HE subgroup was generated based on facial form alone. But because different psychometric measures had been used at different times during patient ascertainment, there was not a common measure available to test the prediction of cognitive impairment from facial dysmorphism. Similar partitions of the Cape Coloured HE subgroup were possible for facial regions not on the mid-line.

While attempting to reproduce the partitioning of the Cape HE subgroup into control-like (HE-HC) and FAS-like (HE-FAS) subsets for the Caucasian cohort, it became apparent that the Cape HE-HC subset displayed orbital hypertelorism. For many years, from an embryological point of view, there has been a suggestion of potential facial form overlap between FAS and the genetic condition DiGeorge syndrome (Sulik, 1986; Lipinski, 2012), This article is protected by copyright. All rights reserved. 
whereby animal models display a phenotype linking alcohol exposure in the 3rd or 4th weeks of human gestation to have FAS-like or DiGeorge-like craniofacial characteristics, respectively. Hypertelorism is a classic feature of DiGeorge syndrome, so it is encouraging to identify orbital hypertelorism in a group of individuals with confirmed heavy alcohol exposure prenatally. However, although it was possible to induce an analogous partition of the HE subgroup in the Caucasian cohort into similar HE-FAS and HE-HC subsets, the Caucasian cohort did not display hypertelorism - only a slight narrowing and upslanting of the periorbital regions.

\section{Discussion}

For the full face, control-FAS discrimination was equally successful in both cohorts. However, differences in discrimination rates for regions of the face suggest that facial form change due to prenatal alcohol exposure may be differently localised and of variable degree in the two cohorts. In particular, the philtrum and profile regions displayed contrasting efficacy in control-FAS discrimination for the two cohorts. Clinicians, of course, do not make diagnoses based on single facial features but it is interesting to consider how less well the philtrum and upper lip vermilion perform in isolation in discriminating between controls and individuals with a FAS diagnosis. To complicate matters, FAS diagnostic criteria permit one of the three facial characteristics to be absent. In the Cape FAS subgroup, chart scores of 4 or 5 for philtrum and lip in the Cape cohort were awarded to $17 / 22(77 \%)$ and $16 / 22$ (73\%) respectively. Corresponding proportions for the Caucasian cohort were $27 / 35$ (77\%) and $30 / 35(86 \%)$, respectively. This partly explains why the philtrum and upper lip vermilion shape based classification rates (Fig. 3B and Table 2) are muted compared to those for the full face or other regions.

This article is protected by copyright. All rights reserved. 
Over and above the variability of the insistence of classic facial characteristics, there is the obvious concern about their recognition in the two cohorts by non-overlapping groups of clinicians. The two cohorts considered here were diagnosed by different clinicians. The Cape Coloured cohort was examined for growth deficits and FAS facial features independently by expert FAS dysmorphologists blinded to prenatal alcohol exposure history, using a standard protocol employing the Astley Lip-Philtrum Guide for assessing philtrum and vermilion, and manual measurement of palpebral fissure length in diagnoses. The Caucasian cohort underwent similar physical examination by one expert dysmorphologist with extensive experience in FAS. In both cases, the same diagnostic criteria were applied. However, the lip-philtrum coding scheme employed for these cohorts is both subjective and categorical and hence is open to misinterpretation and misapplication.

There have been a few studies comparing craniofacial characteristics of FASDs in different ethnicities. Unlike the study reported here, none has considered individuals in our HE category, i.e. with confirmed heavy prenatal alcohol exposure but not meeting clinical diagnosis of FAS or PFAS. Similarly, none of the previous studies employed surface shape analysis and in particular did not consider curvature analysis. Moore et al (2007) evaluated facial features associated with prenatal alcohol exposure in 276 subjects including Cape Coloured individuals (37\%), Finnish Caucasian (36\%), African American (9\%) and North American Caucasian (18\%). Sixteen traditional anthropometric measures were derived from landmark annotations to 3D photographs. For each ethnic group, discriminant analysis identified unique sets of measures supporting control-FAS discrimination rates of $92 \%, 93 \%$, $79 \%$ and $77 \%$ respectively for the four cohorts. The largest and most comprehensive study of ethnic variation in dysmorphic features arising from prenatal alcohol exposure included

This article is protected by copyright. All rights reserved. 
children from Northern Plains Indian communities, South Africa and Italy (May et al, 2010). Unlike the earlier study, this one did consider individuals with a diagnosis of PFAS as well as FAS and controls. A major finding was that approximately $20 \%$ of the variability in core dysmorphology features was associated specifically with ethnicity. Using multilevel analysis, the authors found that the variables predicting dysmorphology across three cohorts were small palpebral fissures, narrow vermilion, smooth philtrum, flat nasal bridge and fifth finger clinodactyly. Specific findings were that South African controls had higher percentages of narrow vermilion, smooth philtrum, short interpupillary distance, flat nasal bridge, and anteverted nostrils, and Northern Plains Indians had higher percentages of railroad track ears. So our findings on the philtrum in Cape Coloured individuals are consistent but we have also been able to use curvature techniques to explicate this difference in more detail.

There are other limitations to our study that need comment. The most obvious limitation is the lack of an African American cohort as a comparison group. At the time of writing, the sample size of this group was too small to produce reliable results. This omission will be addressed in the future. The 3D facial photographs were captured using identical or very similar commercial 3D photogrammetric devices and previous comparisons of such devices have typically shown excellent agreement (Tzou et al, 2014). The cohort size is relatively small, especially the number of individuals with a diagnosis of FAS. Although we used the same number of controls in the age-ethnicity normalisation of facial form, the age distribution in both cohorts is not uniform (Fig. 3). Gaps in contiguity of age will have an effect on the generation of matched running means in the signature normalisation. The small size of the study has also precluded our addressing the effects of sex and body mass index on 3D facial form, although our previous work on facial signatures did take sex into

This article is protected by copyright. All rights reserved. 
account. We have shown that ethnic background differences in underlying facial morphology play a role in the detection of the facial changes induced by prenatal alcohol exposure.

In a study based in the Western Cape of South Africa of two disadvantaged communities (Oelofse, 2002), stunting and underweight was found to be more prevalent in the Cape Coloured infants ( $18 \%$ and $7 \%$, respectively) than in black infants ( $8 \%$ and $2 \%$, respectively). Zinc deficiency was prevalent in 35\% and 33\% of the Cape Coloured and black infants, respectively, although studies in rats have concluded that zinc deficiency is unlikely to affect overall craniofacial growth (Carter, 2012). May et al (2010) have concluded that the effect of under nutrition is a major reason that, for Cape Coloured children, weight, head circumference, and palpebral fissure length remain important predictors of FASD. In a recently recruited Cape Town FASD cohort, maternal nutrition during pregnancy was poor in both the alcohol exposed and control groups (Carter et al., 2014), but there was no evidence of alcohol-related stunting (Carter et al., 2016). Because the groups did not differ in nutritional status in utero, poor nutrition cannot account for the observed effects of alcohol exposure on the facial features. However, research on laboratory animals has shown that differences in nutritional status in utero may modify the effects of prenatal alcohol exposure (Weinberg et al., 1990; Shankar et al., 2006). In our study, we found notable differences in facial dysmorphology caused by prenatal alcohol exposure between the Cape Coloured and Caucasian cohorts related to mid-line and non-mid-line structures. May et al., (2014 and 2016b) addressed the role of nutrition in FASD in the South African population (both in Reproductive Toxicology). In the 2014 article all of the mothers of both FASD and controls were found to be undernourished, but the mothers of children with FASD were more This article is protected by copyright. All rights reserved. 
malnourished especially in calcium, DPA, riboflavin and choline, all nutrients important to growth and development and therefore could influence brain (head) and facial development. So, poor nutrition was indicated in this publication as contributing to FASD feature severity. In the 2016 article, however, all of the mothers were again undernourished, but mothers of children with FASD actually had greater dietary intake of 13 of 25 micronutrients than the mothers of normal controls. Although poor prenatal nutrition may lead to deficits in growth and neurodevelopment, we are unaware of any human or animal studies demonstrating effects of maternal nutrition on dysmorphology outcomes, particularly those seen in FASD. Thus, we believe that prenatal nutritional influences are unlikely to confound or mediate the effects presented in this manuscript. May et al.'s two studies detailed the current diets of women with school-aged children with FASD and made inferences from this about their nutrition during pregnancy. Carter and his colleagues recently conducted a prospective study of diet and anthropometric measures of heavy drinking South African Cape Coloured pregnant women and found similar results (Abstract Carter et al., 2014; manuscript under review); both heavy drinkers and controls had poor gestational weight gain and inadequate dietary intake on a number of nutrients, but there were no clinically significant associations between maternal diet or anthropometry and maternal alcohol intake during pregnancy. Thus, given these recent findings based on contemporaneously-reported dietary and alcohol intake data collected during pregnancy, maternal nutrition is unlikely to confound or mediate FASD dysmorphology outcomes reported here.

This article is protected by copyright. All rights reserved. 
In summary, we have shown how the consideration of facial curvature can assist the recognition of the facial effects of prenatal alcohol exposure and in addition help explain why different facial regions result in inconsistent control-FAS discrimination rates in disparate ethnic groups. In particular, the use of facial curvature has enabled very specific differences to be identified. We have also demonstrated that heavy prenatal alcohol exposure can give rise to orbital hypertelorism, often associated with DiGeorge syndrome. This intriguing result provides supporting evidence for a long-standing suggestion that prenatal alcohol exposure at a particular time, e.g., human gestation week 4, can cause increased separation of the brain hemispheres with a concomitant increase in orbital separation (Parnell et al, 2009; Sulik et al, 1986).

\section{REFERENCES}

Astley SJ, Clarren SK (1996) A case definition and photographic screening tool for the facial phenotype of fetal alcohol syndrome. J Pediatr 129(1):33-41.

Astley SJ, Clarren SK (2000) Diagnosing the full spectrum of fetal alcohol-exposed individuals: introducing the 4-digit diagnostic code. Alc and Alc 35(4):400-410.

Astley SJ, Clarren SK (2001) Measuring the facial phenotype of individuals with prenatal alcohol exposure: correlations with brain dysfunction. Alc and Alc 36(2):147-59.

Bhuiyan Z, Klein M, Hammond P, Haeringen A, Mannens M, Van Berckelaer-Onnes I, Hennekam R (2006) Genotype-phenotype correlations of 39 patients with Cornelia de Lange syndrome: the Dutch experience. Med Gen 43:568-575.

This article is protected by copyright. All rights reserved. 
Carter, R.C., Jacobson, J.L., Molteno, C.D., Jiang, H., Meintjes, E.M., Jacobson, S.W., \&

Duggan, C (2012) Effects of heavy prenatal alcohol exposure and iron deficiency anemia on child growth and body composition through age 9 years. Alcohol Clin Exp Res 36(11):1973-

82.

Carter RC, Duggan C, Bechard L, Molteno CD, Meintjes EM, Jacobson JL, Jacobson SW (2014) Diet and body composition of heavy drinking pregnant women in Cape Town, South Africa. Alcohol Clin Exp Res 38: 251A.

Carter RC, Jacobson JL, Molteno CD, Dodge NC, Meintjes EM, Jacobson, SW (2016). Fetal alcohol growth restriction and cognitive impairment. Pediatrics 138:e20160775

CDC (2004) www.cdc.gov/ncbddd/fasd/documents/fas_guidelines_accessible.pdf

Cox-Brinkman J, Vedder A, Hollak C, Richfield L, Mehta A, Orteu K, Wijburg F, Hammond P (2007) Three-dimensional face shape in Fabry disease. Eur J Hum Gen 15(5):535-542.

Douglas TS, Mutsvangwa TE (2010) A review of facial image analysis for delineation of the facial phenotype associated with fetal alcohol syndrome. Am J Med Genet A 152A(2):52836.

Ellson J, Gansber E, Koutsofios L, North S, Woodhull G (2002) Graphviz- Open Source Graph Drawing Tools. Lecture Notes in Computer Science 2265/2002:594-597.

Fang S, McLaughlin J, Fang J, Huang J, Autti-Rämö I, Fagerlund A, Jacobson SW, Robinson LK, Hoyme HE, Mattson SN, Riley E, Zhou F, Ward R, Moore ES, Foroud T; Collaborative Initiative on Fetal Alcohol Spectrum Disorders (2008) Automated diagnosis of fetal alcohol syndrome using 3D facial image analysis. Orthod Craniofac Res 11(3):162-71.

This article is protected by copyright. All rights reserved. 
Foroud T, Wetherill L, Vinci-Booher S, Moore ES, Ward RE, Hoyme HE, Robinson LK, Rogers J, Meintjes EM, Molteno CD, Jacobson JL, Jacobson SW. (2012) Relation over time between facial measurements and cognitive outcomes in fetal-alcohol exposed children. Alcohol Clin Exp Res 36(9):1634-46.

Gwilliam JR, Cunningham SJ, Hutton TJ (2006) Reproducibility of soft tissue landmarks on three-dimensional facial scans. Eur J Ortho 28(5):408-415.

Hammond P, Hutton TJ, Allanson JE, Campbell LE, Hennekam RCM, Holden S. Murphy KC, Patton MA, Shaw A, Temple IK, Trotter M, Winter RM (2004) 3D analysis of facial morphology. Am J Med Gen 126A (4):339-348.

Hammond P, Hutton TJ, Allanson JE, Buxton B, Campbell L, Clayton-Smith J, Donnai D, Karmiloff-Smith A, Metcalfe K, Murphy KC, Patton M, Pober B, Prescott K, Shaw A, Scambler, Smith ACM, Temple K. Hennekam R, Tassabehji M (2005) Discriminating power of localized three-dimensional facial morphology. Am J Hum Gen 77:999-1010.

Hammond, P (2007) The use of 3D face shape modelling in dysmorphology. Arch Dis Child 92:1120-1126.

Hammond P, Forster-Gibson C, Chudley AE, Allanson JE, Hutton TJ, Holden JJA, Lewis MES (2008) Face-brain asymmetry in autism spectrum disorders. Mol Psych 13: 614-623.

Hammond P, Hannes F, Suttie M, Devriendt K, Vermeesch JR, Faravelli F, Forzano F, Parekh S, Williams S, McMullan D, South ST, Carey J, Quarrell O. (2011) Fine grained facial phenotype-genotype analysis in Wolf-Hirschhorn syndrome, Eur J Hum Gen, in press.

This article is protected by copyright. All rights reserved. 
Hammond P, Suttie M, Hennekam RC, Allanson J, Shore EM, Kaplan FS (2012) The facial signature of Fibrodysplasia Ossificans Progressiva, Am J Med Genet Part A. 158A:1368-1380.

Hammond P, Suttie MJ (2012) Large-scale phenotyping of 3D facial morphology. Hum Mut, 33(5):817-825.

Hoyme HE, May, PA, Kalberg WO, Kodituwakku P, Gossage JP, Trujillo PM, Buckley DG, Miller JH, Aragon AS, Khaole N, Viljoen DL, Lyons Jones K, Robinson LK (2005) A Practical Clinical Approach to Diagnosis of Fetal Alcohol Spectrum Disorders: Clarification of the 1996 Institute of Medicine Criteria. Pediatrics 115(1): 39-47.

Hutton TJ, Buxton BF, Hammond P (2003) Estimating average growth trajectories in shapespace using kernel smoothing. IEEE Trans Med Imag 22:747-753

Jacobson SW, Stanton ME, Molteno CD, Burden MJ, Fuller DS, Hoyme HE, Robinson LK, Khaole N, Jacobson JL (2008) Impaired eyeblink conditioning in children with fetal alcohol syndrome. Alcohol Clin Exp Res 32:365-372.

Jacobson SW, Stanton ME, Dodge NC, Pienaar M, Fuller DS, Molteno CD, Meintjes EM, Hoyme HE, Robinson LK, Khaole N, Jacobson JL. (2011) Impaired delay and trace eyeblink conditioning in school-age children with fetal alcohol syndrome. Alcohol Clin Exp Res $35: 250-264$.

Jacobson SW, Jacobson JL, Stanton ME, Meintjes EM, Molteno CD (2011) Biobehavioral markers of adverse effect in fetal alcohol spectrum disorder. Neuropsychol Rev 21(2):148166.

This article is protected by copyright. All rights reserved. 
Jones KL, Robinson LK, Bakhireva LN, Marintcheva G, Storojev V, Strahova A, Sergeevskaya S, Budantseva S, Mattson SN, Riley EP, Chambers CD (2006) Accuracy of the diagnosis of physical features of fetal alcohol syndrome by paediatricians after specialized training. Pediatrics 118:1734-1738.

Jones KL, Smith DW (1973) Recognition of the fetal alcohol syndrome in early infancy. Lancet 2:999-1001.

Kasperavičiūtė D, Catarino CB, Chinthapalli K, Clayton LMS, ThomM, Martinian L, Cohen H, Adalat S, Bockenhauer D, Pope S, Lench N, Koltzenburg N, Duncan JS, Hammond P, Hennekam RCM, Land JM, Sisodiya S (2011) Uncovering genomic causes of co-morbidity in epilepsy: gene-driven phenotypic characterization of rare micro-deletions. PLOS ONE 6(8):e23182.

Klingenberg C, Wetherill L, Rogers J, Moore E, Ward R, Autti-Rämö I, Fagerlund Å, Jacobson SW, Robinson LK, Hoyme HE, Mattson SN, TK Li TK, Riley EP, Foroud T, and the CIFASD Consortium (2010) Prenatal alcohol exposure alters the patterns of facial asymmetry Alcohol 44(7-8):649-57.

Lipinski RJ, Hammond P, O'Leary-Moore SK, Ament JJ, Pecevich SJ, Jiang Y, Budin F, Parnell SE, Suttie M, Godin EA, Everson JL, Dehart DB, Oguz I, Holloway HT, Styner MA, Johnson GA, and Sulik KK. (2012) Stage-specific ethanol exposure causes unique face-brain dysmorphology patterns in a mouse model of fetal alcohol spectrum disorder. PLOS ONE 7(8): e43067. doi:10.1371/journal.pone.0043067.

May PA, Gossage JP, Smith M, Tabachnick BG, Robinson LK, Manning M, Cecanti M, Jones KL, Khaole N, Buckley D, Kalberg WO, Trujilo PM, Hoyme HE. (2010) Population differences This article is protected by copyright. All rights reserved. 
in dysmorphic features among children with fetal alcohol spectrum disorders. J. Dev. Behav. Pediatr. 31:304-316.

May PA, Hamrick KJ, Corbin KD, Hasken JM, Marais AS, Brooke LE, Blankenship J, Hoyme HE, Gossage JP. (2014) Dietary intake, nutrition, and fetal alcohol spectrum disorders in the Western Cape Province of South Africa. Repr. Toxicol. 46:31-9.

May PA, Marais AS, de Vries MM, Kalberg WO, Buckley D, Hasken JM, Adnams CM, Barnard R, Joubert B, Cloete M, Tabachnick B. (2016a) The continuum of fetal alcohol spectrum disorders in a community in South Africa: Prevalence and characteristics in a fifth sample. Drug Alc. Dep.. 168:274-86.

May PA, Hasken J M, Blankenship J, Marais A S, Joubert B, Cloete M, ... Seedat S. (2016b). Breastfeeding and maternal alcohol use: Prevalence and effects on child outcomes and fetal alcohol spectrum disorders. Repr Toxicol, 63:13-21.

Moore ES, Ward RE, Jamison PL, Morris CA, Bader PI, Hall BD (2001) The subtle facial signs of prenatal exposure to alcohol: an anthropometric approach. J Pediatr, 139(2):215-9.

Moore ES, Ward RE, Jamison PL, Morris CA, Bader PI, Hall BD (2002) New perspectives on the face in fetal alcohol syndrome: what anthropometry tells us. Am J Med Genet, 109(4):249-60.

Moore ES, Ward RE, Wetherill LF, Rogers JL, Autti-Rämö I, Fagerlund A, Jacobson SW, Robinson LK, Hoyme HE, Mattson SN, Foroud T; CIFASD. (2007) Unique facial features distinguish fetal alcohol syndrome patients and controls in diverse ethnic populations. Alcohol Clin Exp Res, 31(10):1707-13.

This article is protected by copyright. All rights reserved. 
Mutsvangwa T, Douglas TS (2007) Morphometric analysis of facial landmark data to characterize the facial phenotype associated with fetal alcohol syndrome. J Anat, 210(2):209-20.

Mutsvangwa TE, Meintjes EM, Viljoen DL, Douglas TS (2010) Morphometric analysis and classification of the facial phenotype associated with fetal alcohol syndrome in 5- and 12year-old children. Am J Med Genet A 152A(1):32-41.

Oelofse A, Van Raaij JM, Benadé AJ, Dhansay MA, Tolboom JJ, Hautvast JG. Disadvantaged black and colored infants in two urban communities in the Western Cape, South Africa differ in micronutrient status. Public Health Nutr. 2002;5(2):289-94.

O'Leary CE, Thomas KGF, Molteno CD, Jacobson JL, Jacobson SW (2011) Verbal learning and memory in fetal alcohol spectrum disorder: Findings from Cape Town and Detroit. Alcohol Clin Exp Res, 35(s1):111A.

Parnell SE, O'Leary-Moore SK, Godin EA, Dehart DB, Johnson BW, Allan Johnson G, Styner MA, Sulik KK (2009) Alcohol Clin Exp Res 33(6):1001-11.

Roozen S, Peters G-JY, Kok G, Townend D, Nijhuis J and Curfs L. (2016) Worldwide Prevalence of Fetal Alcohol Spectrum Disorders: A Systematic Literature Review Including Meta-Analysis. Alcohol Clin Exp Res, 40: 18-32.

Shankar K, Hidestrand M, Liu X, Xiao R, Skinner CM, Simmen FA, Badger TM, Ronis MJ. (2006). Physiologic and genomic analyses of nutrition-ethanol interactions during gestation: Implications for fetal ethanol toxicity. Exp Biol Med 231(8):1379-1397.

This article is protected by copyright. All rights reserved. 
Sturn A, Quackenbush J, Trajanoski Z (2000) Genesis: cluster analysis of microarray data.

Bioinformatics (2002) 18 (1): 207-208.

Sulik KK, Johnston MC, Daft PA, Russell WE, Dehart DB. Fetal alcohol syndrome and

DiGeorge anomaly: critical ethanol exposure periods for craniofacial malformations as

illustrated in an animal model. Am J Med Genet Suppl. 1986;2:97-112.

Suttie M, Foroud T, Wetherill L, Jacobson JL, Molteno CD, Meintjes EM, Hoyme HE, Khaole N, Robinson LK, Riley EP, Jacobson SW, Hammond P (2013) Facial dysmorphism across the fetal alcohol spectrum. Pediatrics 131 (3), e779-e788.

Tassabehji M, Hammond P, Karmiloff-Smith A, Thompson P, Durkin ME, Thorgeirsson S, Metcalfe K, Rucka A, Hutton T, Hogan T, Stewart H, Read A, Maconochie A, and Donnai D (2006) GTF2IRD1 in craniofacial development of humans and mice. Science 310:1184-1187.

Tobin JL, DiFranco M, Eichers E, May-Simera H, Garcia M, Yan J, Justice M, Briscoe J, Mayor R, Lupski JR, Hammond P, Beales PL (2008) Defects of Shh transduction and neural crest cell migration underlie craniofacial dysmorphology in Bardet-Biedl syndrome. PNAS 105(18):6714-9.

Tzou C-HJ, Artner NM, Pona I, Hold A, Placheta E, Kropatsch WG, Frey M. (2014) Comparison of three dimensional surface-imaging systems. J Plast Reconstr Aesthet Surg 67:489-497.

Weinberg J, D'Alquen G, Bezio S (1990). Interactive effects of ethanol intake and maternal nutritional status on skeletal development of fetal rats. Alcohol 7(5):383-388.

This article is protected by copyright. All rights reserved. 


\section{Figure Legends}

\section{Figure 1}

Landmark annotations $(n=24)$ and regions of the face analysed $(n=8)$

Mid-line landmarks ( $n=6)$ : nasion; pronasale; subnasale; upper/lower lip centres; gnathion

Paired left-right landmarks ( $n=9)$ : lower ear attachment; tragion; inner \& outer canthion; upper \& lower mid-eyelid; alare; cheilion; cupid's bow.

Facial regions ( $n=8)$ : face, eyes, philtrum, mandible, nose, malar, upper lip vermilion, profile.

\section{Figure 2}

Collapsed groove signature graph for the philtrum for all FAS individualsThe greater homogeneity of the Caucasian philtrum groove signatures is well delineated in the individual heat maps and is confirmed quantitatively in the collapsed signature graph by the large subcluster of 32 of 35 Caucasian individuals with FAS and the dispersion indices: Caucasian (0.10) and Cape Coloured (0.54).

\section{Figure 3}

Facial growth (PCA1) for control, FAS and HE subgroups of each cohort: A) Cape Coloured; B) Caucasian.

\section{Figure 4}

A. Box plots for age-ethnicity normalized PCA1 values reflecting overall facial growth B. Comparison of mean classification results in supplementary table ST1 using a simpler visualisation (mean of CM, LDA and SVM) for both cohorts for each facial region considered. Consistency of classification in the two cohorts is lower for mid-line regions (nose, profile, philtrum and lip vermilion) and higher in non-mid-line regions (face, eyes, mandible and malar region).

This article is protected by copyright. All rights reserved. 


\section{Figure 5}

Shape, normalised curl and groove of the mean philtrum of Caucasian/Cape FAS subsets (deepest red < -1.0 st dev; deepest blue $>1.0$ st dev)

\section{Figure 6}

Signature graphs for FAS (red circles), HE who are FAS-like (HE-FAS) in displacement facial difference from controls (green squares) and $\mathrm{HE}$ who are control-like (HE-HC) in their difference from matched controls (green circles): A) Cape Coloured; B) Caucasian.

\section{Figure 7}

Orbital hypertelorism in Cape Coloured HE subgroup with more control-like facial dysmorphism (HE-HC).

Signature of average of Cape Coloured HE-HC subgroup normalised against age-ethnicity matched controls (A). Box plots comparing FAS, controls (HC), and subpartitions of HE into HE-FAS and HE-HC for inner canthal separation (B), inter-pupillary distance (C) and outer canthal separation (D)

This article is protected by copyright. All rights reserved. 


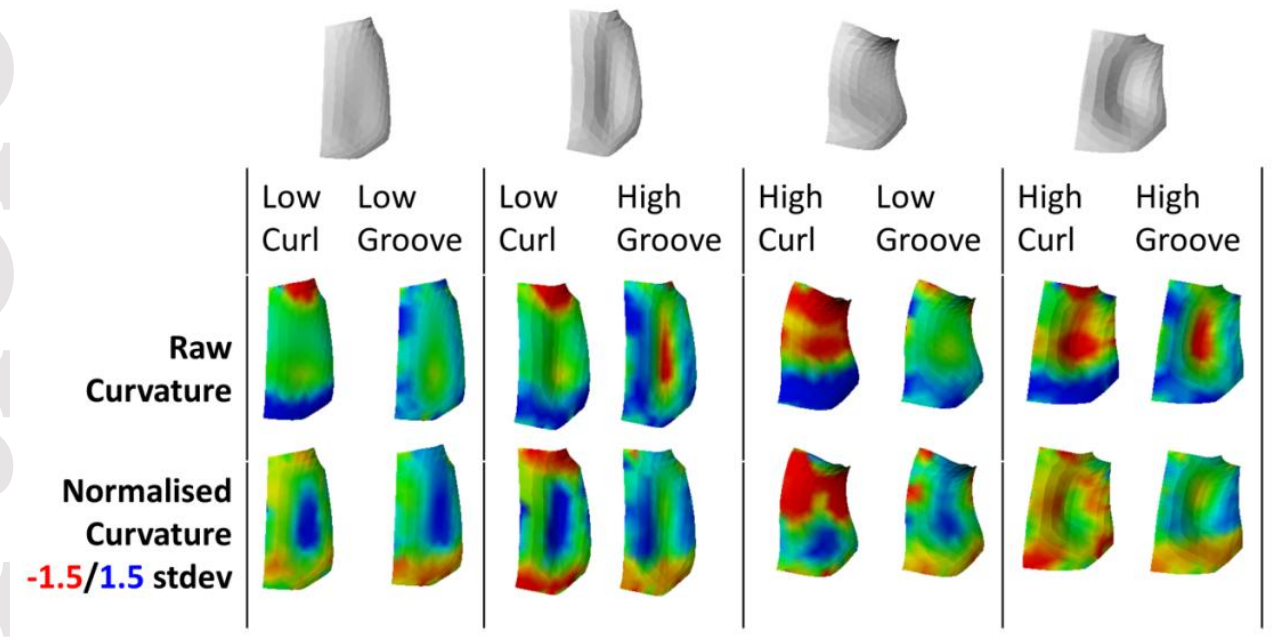

Table 1

This article is protected by copyright. All rights reserved. 


\begin{tabular}{|c|c|c|c|c|c|c|c|c|c|c|c|c|c|}
\hline & & \multicolumn{2}{|c|}{ SEX } & \multicolumn{2}{|c|}{ AGE } & \multicolumn{2}{|c|}{ BMI } & \multicolumn{2}{|c|}{ PFL } & \multicolumn{2}{|c|}{ VERMILION } & \multicolumn{2}{|c|}{ LIP } \\
\hline & & $\mathrm{F}$ & $\mathrm{M}$ & MEAN & SD & MEAN & SD & MEAN & SD & MEAN & SD & MEAN & SD \\
\hline \multirow[t]{2}{*}{$\mathrm{HC}$} & CAPE & $50.7 \%$ & $49.3 \%$ & 10.1 & 2.6 & 18.4 & 4.1 & 2.6 & 0.4 & 3.1 & 0.6 & 2.9 & 0.6 \\
\hline & CAUC & $41.1 \%$ & $58.9 \%$ & 11.5 & 3.2 & 19.2 & 3.9 & 2.6 & 0.3 & 3.0 & 0.5 & 3.0 & 0.4 \\
\hline \multirow[t]{2}{*}{ FAS } & CAPE & $45.5 \%$ & $54.5 \%$ & 10.6 & 2.9 & 15.7 & 1.6 & 2.4 & 0.2 & 3.8 & 0.7 & 4.0 & 0.7 \\
\hline & CAUC & $60.0 \%$ & $40.0 \%$ & 11.8 & 3.4 & 16.4 & 2.4 & 2.3 & 0.2 & 3.9 & 0.6 & 3.9 & 0.6 \\
\hline \multirow[t]{2}{*}{$\mathrm{HE}$} & CAPE & $52.0 \%$ & $48.0 \%$ & 10.4 & 2.7 & $17.1^{*}$ & 2.7 & 2.6 & 0.2 & 3.1 & 0.8 & 3.0 & 0.8 \\
\hline & CAUC & $39.7 \%$ & $60.3 \%$ & 12.1 & 3.0 & 18.9* & 4.4 & 2.5 & 0.2 & 3.1 & 0.5 & 4.0 & 0.5 \\
\hline
\end{tabular}

Table 2

This article is protected by copyright. All rights reserved. 
Facial region

Face

Profile

Eyes

Malar

Mandible

Nose

Lip vermillion

Philtrum

Table 3
$\mathrm{CM}$

LDA

\begin{tabular}{cc} 
Cape & Cauc \\
\hline 0.96 & 0.95 \\
0.95 & 0.83 \\
0.95 & 0.92 \\
0.94 & 0.90 \\
0.88 & 0.86 \\
0.86 & 0.94 \\
0.73 & 0.70 \\
0.70 & 0.83
\end{tabular}

\begin{tabular}{cc} 
Cape & Cauc \\
\hline 0.96 & 0.96
\end{tabular}

0.96

0.95

0.82

0.93

0.91

0.88

0.94

0.69

0.85
SVM

\begin{tabular}{cc} 
Cape & Cauc \\
\hline 0.98 & 0.96 \\
0.94 & 0.86 \\
0.94 & 0.92 \\
0.95 & 0.91 \\
0.93 & 0.85 \\
0.89 & 0.95 \\
0.84 & 0.73 \\
0.76 & 0.90
\end{tabular}

FACE

\begin{tabular}{|c|c|c|c|c|c|c|c|c|}
\hline & \multicolumn{4}{|c|}{ FACE } & \multicolumn{4}{|c|}{ PHILTRUM } \\
\hline & \multicolumn{2}{|c|}{ Displacement } & \multicolumn{2}{|c|}{ Curvature } & \multicolumn{2}{|c|}{ Displacement } & \multicolumn{2}{|c|}{ Groove } \\
\hline & Caucasian & Cape & Caucasian & Cape & Caucasian & Cape & Caucasian & Cape \\
\hline CONTROLS & $0.80 \pm 0.03$ & $0.69 \pm 0.06$ & $0.58 \pm 0.02$ & $0.77 \pm 0.02$ & $0.69 \pm 0.02$ & $0.79 \pm 0.01$ & $0.19 \pm 0.02$ & $0.59 \pm 0.02$ \\
\hline FAS & $0.76 \pm 0.02$ & $0.50 \pm 0.03$ & $0.70 \pm 0.01$ & $0.28 \pm 0.03$ & $0.56 \pm 0.02$ & $0.75 \pm 0.02$ & $0.07 \pm 0.01$ & $0.55 \pm 0.04$ \\
\hline
\end{tabular}

Table 4

This article is protected by copyright. All rights reserved. 
TABLE LEGENDS

Table 1

Examples of philtrum curl and groove curvature, both raw and normalised

row 1: surface of philtrum; row 2: degree of curl and groove; row 3: raw curvature; row 4: curvature normalised against age-ethnicity matched controls (red < -1.5 SD; blue > 1.5 SD)

Table 2

Pairwise Cape-Coloured and Caucasian cohort comparisons of HC, FAS and HE subsets

The only significant difference was between HE subsets for BMI $\left({ }^{*} p<0.01\right)$

Table 3

Agreement of clinical diagnosis and control-FAS face discrimination using surface displacement (estimated from area under ROC curves as probability of correct classification of random pair of control and FAS faces) $\mathrm{CM}=$ closest mean; LDA=linear discriminant analysis; SVM=support vector machines

Table 4

Average Caucasian/Cape Coloured dispersion indices and 95\% $\mathrm{Cl}$ for normalised displacement and curvature.

The first pair of emboldened indices $(\mathbf{0 . 5 0}$ and $\mathbf{0 . 2 8})$ in the FAS row and counterparts in the CONTROLS row (0.69 and 0.77) suggests that prenatal alcohol exposure reduces signature graph dispersion and makes the Cape Coloured face more homogeneous in its difference This article is protected by copyright. All rights reserved. 
from controls. Similarly, the second pair (0.56 and 0.07 ) compared to counterparts (0.69 and 0.19) suggests that prenatal alcohol exposure makes the Caucasian philtrum groove curvature more homogeneous in its difference from controls.
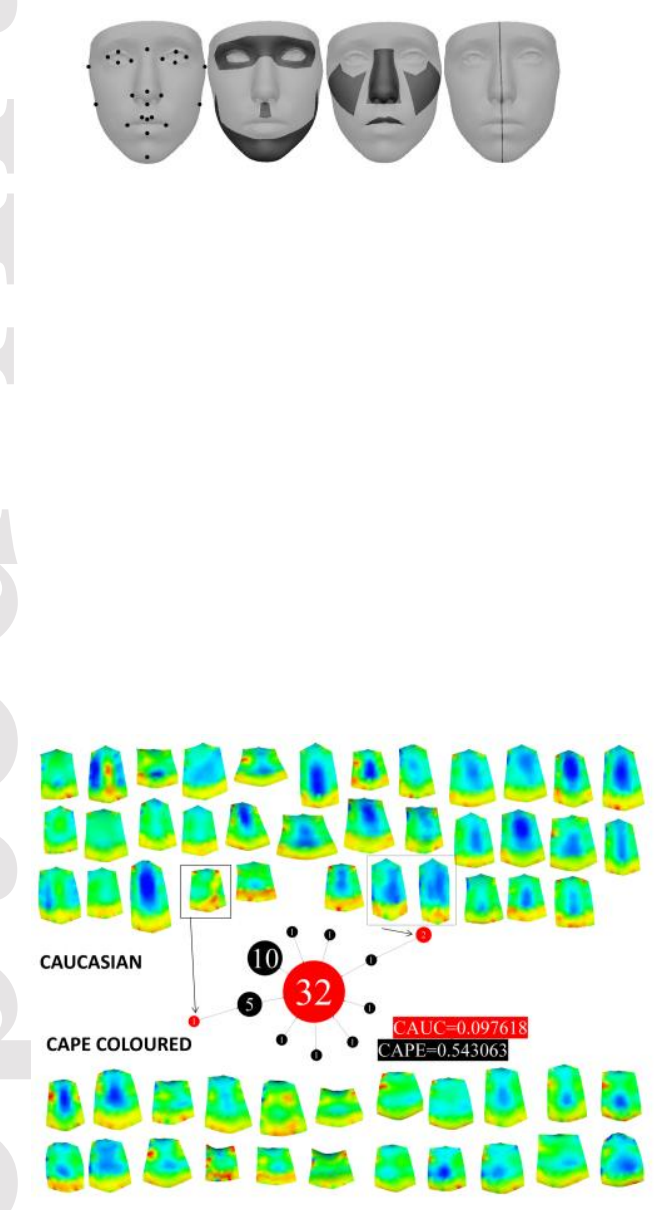

This article is protected by copyright. All rights reserved. 

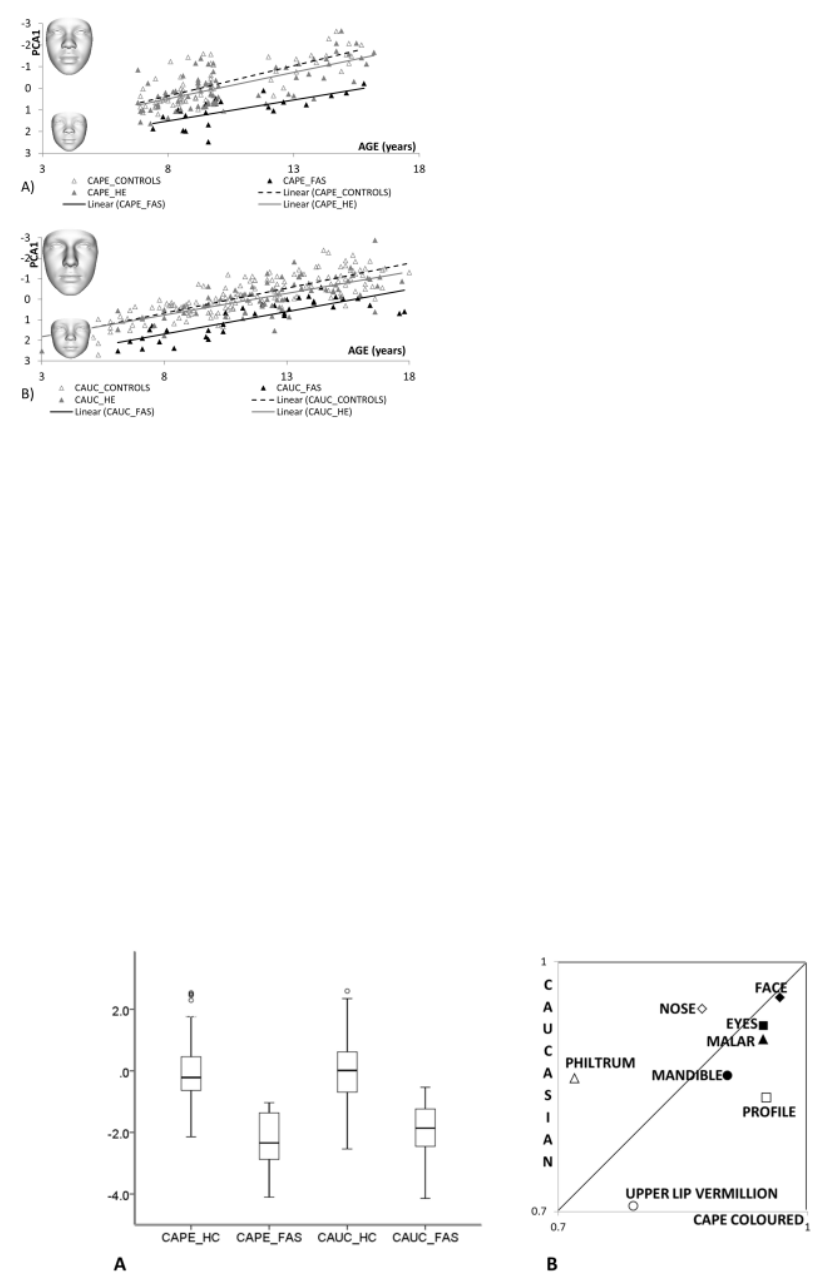

This article is protected by copyright. All rights reserved. 

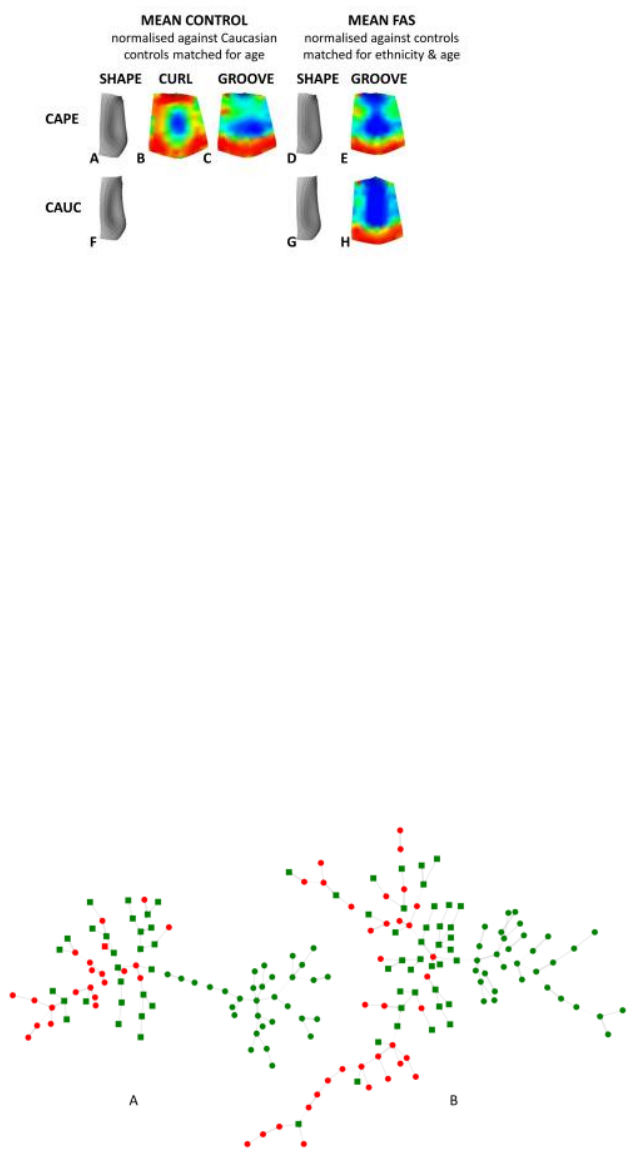

This article is protected by copyright. All rights reserved. 

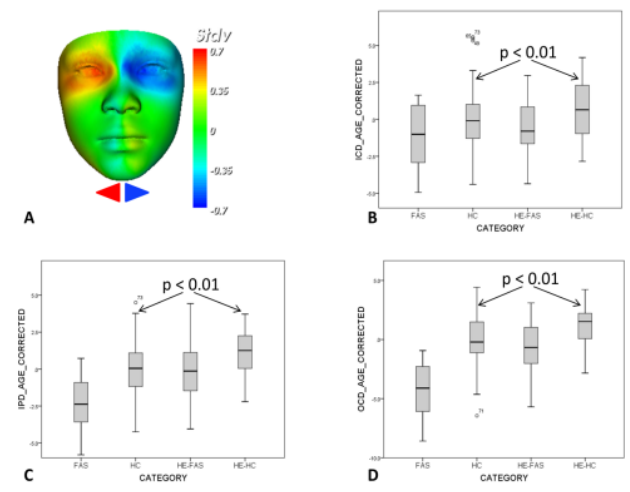

This article is protected by copyright. All rights reserved. 